\title{
DISCURSO PRONUNCIADO POR EL DR. JAIME A. VIÑAS ROMAN, RECTOR DE LA UNIVERSIDAD NACIONAL PEDRO HERIQUEZ UREÑA, EN LA APERTURA DEL 3er. CONGRESO LATINOAMERICANODEFITOPATOLOGIA, EL DIA 19 DE AGOSTO DE 1985
}

Señoras y Señores:

Es un honor $y$ un privilegio dirigirme a esta selecta audiencia en la apertura del 3er. Congreso Latinoamericano de Fitopatología. Asimismo hoy celebramos concurrentemente el $1 \mathrm{er}$. Congreso de la Sociedad Fitosanitaria dominicana. Me hago eco de las palabras de su Presidente, el Ingeniero Agrónomo Germán Vásquez, y de los distinguidos oradores que me han precedido, para darles a ustedes la bienvenida a nuestro país, con el deseo de que, no sólo enriquezcan sus intelectos con las ponencias $y$ talleres técnicos, sino también aprovechen para disfrutar de la belleza y hospitalidad de nuestro pueblo dominicano. Una vez más, pues, sean todos bienvenidos a su otro hogar en República Domínicana.

Permítanme empezar mis palabras con una nota mitad en broma y mitad en serio. Ya que todos ustedes son especialistas, técnicos $y$ consultores en el campo de la fito- patología, de las ciencias del agro, $u$ otras disciplinas afines, el cuento que haré viene, quizás, como anillo al dedo.

El Profesor Alberto Moncada, uno de los más famosos planificadores sociales y educativos de América Latina, cuenta un chiste que circulaba profusamente por las comidas, cenas y congresos de esa que él llama la "Mafia intelectual" que forman los consultores, asesores y expertos. En los años del desarrollismo -cuenta él- un ingeniero agrónomo viajaba por cuenta de la FAO por un país del altiplano andino. Amigo de recorrer concienzudamente el campo, se metió un día por caminos que no conocía y se le hizo de noche en una pequeña aldea. Detuvo su carro en la plaza y comenzó a llamar a las puertas buscando posada.

La primera mujer que le abrió, se negó a darle hospedaje porque no estaba su marido. El ingeniero trató de tranquilizarla. "Yo soy, señora, un experto de la FAO y mi trabajo consiste en ayudar a los 
agricultores $y$ ganaderos a tener mejores rendimientos. Puede fiarse de mí." Pero ni por esas. La buena india le dió con la puerta en las narices. Una y otra vez recibió el mismo trato, hasta que al final una mujer basó su negativa en que sólo tenía una cama. El ingeniero vió el cielo abierto y le dijo: "Señora, no tiene nada que temer de mí. Yo soy experto de la FAO, etc., etc." La mujer, una cuarentona de buen ver, que criaba ella sola animales domésticos, le dió albergue y nuestro ingeniero pasó la noche a buen recaudo. A la mañana, siguiente, cuando ya se marchaba, pasó por la pequeña chacra, detrás de la casa, donde la dueña criaba sus aves, y vió que en un corralito tenía cinco gallinas y cinco gallos. Queriendo pagar con su asesoramiento la buena acogida, ya que la mujer no había querido cobrarle, le dijo: "Señora, el ciclo reproductor de estos animales sólo requiere un gallo por cada cinco gallinas, de modo que le sobran cuatro." Pero ella contestó: "Mi señor ingeniero, es que sólo tengo un gallo que sea verdaderamente gallo. Los otros son -concluyó- expertos de la FAO".

El chiste viene a cuenta porque, en estos tiempos que corren los técnicos, especialistas y consultores de todo tipo tienen una creciente sensación de que su participación en la dinámica histórica es tan limitada y ha sido tan descalificada al menos como la de aquel experto de la FAO.

Ciertamente, después de unos años gloriosos allá por los 60 , pocos y ya lejanos, los especialistas en educación, en planificación, en urbanismo, en reforma agraria, etc., iban y venían, de conferencia en conferencia, de congreso en congre- so $y$ de país en país, dispensando su sabiduría, y muchas veces sus buenas intenciones y profusos estudios, se han ido esfumando ante duras $y$ dolorosas realidades poirticas, culturales y económicas de nuestra América Hispana.

Afortunadamente, el ser humano no desiste en su empeño de avanzar y perfeccionarse. Este Congreso es una muestra significativa, y la presencia de ustedes evidencia un acto de fe en el conocimiento, en la investigación y en la educación de los pueblos. Yo siento una sincera y profunda admiración por las personas que dedican su vida $y$ su trabajo a expandir nuestro conocimiento de la naturaleza de la tierra, de las plantas, de los animales. Naturalmente, durante varias décadas he estado intimamente vinculado a estas ramas especiales de las ciencias y las tecnologías, ya sea en mi profesión de Médico Veterinario, así como de Profesor de Ciencias y Decano de la Facultad de Ciencias, y ahora como Rector de la Universidad Nacional Pedro Henríquez Ureña.

Pero mi admiración hacia ustedes no nace sólamente de mis gustos o preferencia vocacionales, ni siquiera, incluso, de la enorme importancia que tiene el trabajo que ustedes realizan. Más adelante hablará sobre ello. Mi admiración surge de razones muy profundas, que son, en rigor, de tipo filosófico, o si me dispensan un término altisonante, hay motivos "cosmológi$\cos ^{\prime \prime}$, que nos mueven a dar aprecio, valor y admiración al trabajo de un fitopatólogo.

Déjenme explicarme, por unos minutos, pues es un asunto sobre el cual poco o casi nada se reflexiona, especialmente en con- 
gresos como éste, que tiene un carácter muy especializado y un campo muy definido de indagación.

Se trata, ni más ni menos, que de la antigua y arcana sabiduría del viejo Mediterraneo y de la cuenca del Nilo, con la famosa sentencia de Hermes Trismegistus de: "tal como es arriba, es abajo, el Microcosmos y el Macrocosmos son uno".

Para el científico contemporáneo, esta es una de las máximas más fecundas y enigmáticas. Quiero ponderar unas ideas sobre esto, pues nos concierne a todos, y más aún a los hombres $y$ mujeres que hacemos de la ciencia y de la educación nuestro quehacer vital. Además, creo que puede ser algo novedoso, o acaso inusitado, abordar el trabajo de un fitopatólogo desde otras perspectivas y latitudes conceptuales. Veamos esto.

No se si recuerdan ustedes, allá a mediados de la década del 50, una interesantísima y fascinante película de ciencia ficción sobre un hombre que, por razones oscuras $y$ que se ignorabán, se iba reduciendo de tamaño cada día. Era un hombre normal y corriente, con su esposa e hijos, y empleado de una oficina. De repente, un día cuando se prepara para irse para su empleo, en la mañana, encuentra que la ropa le queda holgada y larga. Pantalones y camisa no le sirven. Ahí empieza una dolorosa, dramática y patética odisea. Cada día se encogía en pulgadas y centímetros. Su existen. cia diaria se iba convirtiendo en un verdadero calvario de burlas, de incomprensión, de aprender a subsistir, hasta durmiendo dentro de una caja de fósforo que su esposa le puso en el suelo de la habitación.

Imagínense ustedes a este in- feliz sujeto, reduciéndose progresiva e inconteniblemente en su tamaño, sufriendo y padeciendo los tropiezos más fantásticos e imaginables. Hasta que un día, sale al jardín, en una hermosa mañana de primavera, y se mete por dentro de uno de los huecos o rendija de una tela metálica pequeñísima, y mirando hacia arriba ve la hoja de una planta. La hoja era muy pequeña, pero para él era ciertamente de un tamaño casi inmensurable, y en la hoja había una pequeña gota del rocío de la mañana, que le parecía como un vasto $y$ enorme océano, y él se da cuenta, en ese maravilloso instante y ante ese majestuoso espectáculo de la gota y de la planta, que iba a llegar un momento en que su tamano físico se haría infinitesimal.Entoncesdecidió que su profunda angustia y el terror psicológico de desaparecer en la nada, era una ilusión, una ficción. Llegaría un momento en que se uniría en su vida lo más grande y lo más pequeño. Se operaría una misteriosa unión entre el Macrocosmos del universo y su propio Microcosmos infinitesimal.

Señores, esta película, como toda obra de arte en ciencia-ficción, plantea interesantímas cuestiones en la física, la matemática, la filo. sofía y hasta la teología. No la despachemos, de reojo, muy a la ligera.

Porque precisamente nos debe interesar, como hombres de estudio y de cienciaa la aventura en el flujo del pensamiento humano que nos coloca en el albor de un nuevo siglo, un siglo que en pala. bras de Theilard de Chardan, será el de "Nuestra Conciencia en Expansión."

Una de las hipótesis de la cosmología moderna sobre el origen del universo consiste en suponer 
que éste se encuentra en auge entre una explosión inicial y una expansión ilimitada. Nuestra minúscula tierra ý nosotros mismos, más minúsculos todavía, estaríamos así metidos en la misma inmensa aventura.

Es en esta aventura del pensamiento, de la inventiva $y$ de la imaginacióm, donde nuestra película mencionada cobra su plena fecundidad: la expansión simultánea hacia lo más grande y hacia lo más pequeño. Esta es una de las características más notables de la época: la expansión del conocimiento humano tanto hacia el macrocosmos como hacia la naturaleza primordial que nos da la vida $y$ el sustento.

Por un lado, asistimos a los fantásticos descubrimientos de la astrofísica y la astronomía. Con potentes radiotelescopios ya podemos detectar señales procedentes de estrellas situadas a cinco mil millones de años luz respecto a la tierra, es decir, señales que tardan cinco mil millones de años en llegar hasta nosotros, a pesar de su enorme velocidad de propagación, de más de mil millones de kilómetros por hora. Pues bien, esto no es todo ya que a estas tremendas distancias hay que añadir un dato adicional: todas esas estrellas parecen estar alejándose de nosotros, y sus velocidades de desplazamiento se aproximan a la de la luz. Cuando uno se imagina esas acumulaciones de varios millones de estrellas, que denominamos galaxias, con una masa total del orden de los 100,000 millones de veces superior a la del sol, lanzadas a una velocidad que, en conjunto puede aproximarse a los 1,000 millones de kilómetros por hora, ¿acaso no rebasa la misma imaginación?.

La aventura de nuestro pensamiento macrocósmico es sencillamente fascinante. $Y$ tambien fascinantes es la otra aventura, en la física, la biología, la botánica, la genética, que penetra en el terreno de lo infinitamente pequeño, en las estructuras, componentes y relaciones más sutiles de la materia física, vegetal y biológica.

De este modo, el hombre de nuestra época, ha podido situarse materialmente en relación al conjunto de su habitáculo. Es aquí, precisamente donde me parece que este Congreso tiene su más radical significación. Sin lugar a du da s ustedes harán avances importantes en determinar, con precisión, tipos de enfermedades de las plantas, su origen, su contral y su prevención. Discutirán sobre técnicas y metodologías especiales, equipos adecuados, costos y viabilidad. Pero todo esto, en primera y última instancia, tiene un sentido mayor, y les decía que tenía una subyacente razón cosmológica.

En efecto, ya pueden entender, como señalaba hace unos minutos, por quése puede ver la fitopatología con una óptica diferente, humana y filosófica.

Creánme, que este es un Congreso muy importante. Se lo dice quien no es experto en fitopatología. Les diré, también, por qué se les debe respetar y admirar.

Porque ustedes están seriamente empeñados en hacer que 
nuestra tierra, nuestro habitat, sea habitable. El fitopatólogo es uno de los más importantes pioneros en esta aventura de la ciencia, la tecnología y la cultura moderna.

Por eso mi país y toda Améri$\mathrm{ca}$, debe sentir con expectativa $y$ orgullo, la presencia de estudiosos como ustedes.

La fitopatología no es, en rigor, la ciencia que estudia la enfermedad de las plantas o su control. Es eso, sT, pero ustedes se definen ${ }^{\circ}$ primordialmente por su amor a la tierra, a la naturaleza. Claro, esto les puede sonar a romanticismo, o a poesía, y no me disculpo por ello. De eso, más que nada, es de lo que necesitamos. Seres humanos que con su tesón intelectual, con su disciplina científica, se hagan amantes de la tierra, se hagan sensibles a la belleza, al orden natural de la vida, al ritmo biológico y ecológico de las cosas en este habitat que es nuestro planeta tierra.

En toda la humanidad vuelve siempre a encontrarse el vivo deseo $y$ el ferviente anhelo de regresar a la madre tierra. Este anhelo casi no se distingue del de la muerte. La tierra es una mujer y la mujer una tierra. Por eso desde tiempos inmemoriales, el ser humano se ha ocupado sobre todo de la madre $y$ de la tierra, como lo demuestran los que están familiarizados con la agricultura, con el folklor campesino, con la antropología y la literatura.

El viejo de los "Canterbury Tales" de Chaucer, que no puede morir, suspira:

"Cual prisionero inquieto, por la tierra -puerta que me condu- ce hacia la madre- camino, y con mi báculo, más tarde o más temprano golpeo y digo: dejame entrar, madre querida."

Este grito de "déjame entrar" también fue el estímulo de Bacon, en su afán de penetrar en los secretos de la naturaleza, viéndola, como él, tal cual "libro abierto".

Es así, mis apreciados colegas, como en este Congreso, se deben hacer penetrantes esfuerzos por desentrañar el conocimiento de este universo material, biológico y vegetal, que nos haga vivir una existencia más enriquecedora, humana, armónica y saludable.

Pero, como ustedes saben bien, no todo es poesía y flor en la viña del señor: tenemos grandes escollos que vencer. En este Congreso tienen que hacerse planteamientos serios y rigurosos, pues nos acosan problemas que debemos resolver.

Alguien me puede decir: "Pero, Señor Rector, son tantas las cosas que aún no sabemos en materia de fitopatología, falta mucho por experimentar y verificar", etc., etc..., y yo le responderé: "De acuerdo, es mucho lo que no se sabe, pero también ya tenemos investigaciones $y$ conocimientos empíricos de asuntos, que sí sabemos y que sí podemos aplicar."

Estoy seguro de que ustedes ya disponen de ideas, técnicas, aplicaciones y recursos, que pueden seguir beneficiando a este planeta nuestro. Pero a estas alturas ya ustedes deben haber comprendido que la fitopatología es una zona blanda de la investigación y del co- 
nocimiento puro $y$ aplicado, que está muy condicionada por esas zonas duras que son la política partidista, los intereses económicos $y$ las actividades humanas egoistas $y$ recalcitrantes $y$ a veces hasta faltas de la moralidad que debe pautar todas las actividades de los hombres.

Estas son realidades que debemos plantear abierta $y$ valientemente. Ustedes tienen que hacer un llamado para que en nuestros países se maneje el control fitosanitario con criterios estrictamente profesionales, científicos y técnicos. iBasta ya de intromisiones indebidas, de personas sin escrúpulo, amorales, en cuestiones $\tan$ serias y $\tan$ delicadas como la producción de alimentos y la salud de un pueblo.

La política partidista y el manejo inescrupuloso y fraudulento de los fondos económicos (públi$\cos y$ de agencias internacionales), nos hacen daños a veces irreparables, o que tardan generaciones en poder ser corregidos. Asimismo, la ignorancia, la indiferencia y la apatía ante el reclamo justo y legitimo de sus recomendaciones fitosanitaria, han provocado enfermedades y han perturbado cosechas, que con mejores criterios éticos y científicos, pudo haberse corregido.

La tierra, la madre tierra, es un símbolo y es también una realidad. No se puede jugar con la naturaleza. Nuestros países deben aprender rápidamente a tomarse en serio las condiciones materiales del habitat. Esta toma de conciencia, me parece, la están llevando a cabo, mayormente, las instituciones edu- cativas universitarias, los centros de investigación adscritos a universidades o empresas privadas, $y$ algunas agencias gubernamentales. Este Congreso puede ayudar a desempolvar la ignorancia y la dejadez de muchas personas $y$ agencias, en torno a la sanidad vegetal como prioridad indispensable en nuestra política pública y privada.

Si me permiten, quiero hacer alusión a ciertos problemas y prioridades que deben ser atendidos, con seriedad, honestidad y criterio profesional, por el Gobierno de la República Dominicana. Menciono estos puntos como fruto de muchos años en los que me he dedicado día a día a la docencia y a la dirección en el campo de la Ciencia, la Salud; la Agricultura y la Educación. - Lo digo con ánimo de que nuestra vida sea mejor, que amenos y respetemos esta bendia tierra que nos ofrece el sustento y nos nutre.

En primer lugar, nos estamos enfrentando con serios problemas cuarentenarios que debemos ya resolver. Sabemos que desde el punto de vista de la cuarentena interna, se deben implementar cordones fitosanitarios que eviten el paso de material vegetativo procedentes de áreas en donde se hayan detectado alguna enfermedad de importancia económica, con fines de impedir la introducción de las mismas a zonas libres del problema.

Con relación a la cuarentena externa, creo que se debe dar mayor apoyo económico a la apertura de la Estación Cuarentenaria de Post-entrada y Sub-estaciones. Esto tiene por finalidad poseer un 
mayor control de la importación de material vegetativo y sus derivados para evitar la introducción de pestes y enfermedades que puedan incidir negativamente en nuestra agricultura. No nos cansaremos de decir, una y otra vez, el que ya es hora de que se respeten y ejecuten las decisiones tomadas por los técnicos especializados en las áreas de Sanidad y Protección Vegetal, basadas en los resultados de análisis realizados en los laboratorios de instituciones acreditadas y de que personas ineptas e incapaces hagan decisiones importantes con base únicamente de sus prerrogativas políticas.

Un asunto de enorme prioridad, y del cual quiero hacerme eco junto a otros investigadores, es que se tomen pasos concretos y rápidos para crear el IDIA. Este Congreso puede dar apoyo en el sector gubernamental para agilizar la creación del Instituto Dominicano de Investigaciones Agropecuarias (IDIA) en el cual se unificarán recursos económicos y técnicos para investigar (entre otros) problemas causados por enfermedades en cultivos económicos, estimar sus pérdidas y buscar soluciones adaptadas a nuestro medio, en un corto, mediano y largo plazo. También se necesita capacitar más personal técnico a nivel de post-grado, en las áreas de Protección Vegetal y además reforzar los Programas de Extensión y Capacitación para que se agilice la divulgación de la tecnología generada por la investigación.

Una sexta recomendación que me parece recoge el sentir de algu- nos de ustedes, es que se deben reducir los impuestos que gravan los pesticidas (específicamente fungicidas) utilizados en cultivos como arroz, habichuela, plátano y hortalizas, así como equipos de fumigación. El alivio económico que esto representa constituirá un gran estímulo para nuestra productividad agrícola. El Gobierno Dominicano debe atender y vigilar la producción de semillas que estén libres de enfermedades. En este sentido debe darse apoyo $y / 0$ facilidades al sector público y privado en la implementación de una politica tendiente a producir suficientes semillas libres de enfermedades que satisfagan la demanda nacional para evitar la importación en este renglón.

Señores, no me parece que estoy pidiendo que lleguemos a Plutón, ni que el Gobierno nos traiga una estrella. Estas son consideraciones razonables, viables y sensatas. Nosotros en la Universidad Nacional Pedro Henríquez Ureña, desde hace tiempo estamos luchando por darle a este país, sin ninguna ambición personal ni institucional, una educación altamente científica y técnica en el Campo Agropecuario. La UNPHU, que co-auspicia este importante Congreso, está muy consciente de la importancia que tiene la Fitopatología en el desarrollo agrícola nacional y por ello esta rama de las ciencias agropecuarias constituirá una de las bases importantes de la Universidad Agraria que nuestra institución está desarrollando en el Recinto Agropecuario y Finca Experimental de Nigua. $Y$ 
más en estos momentos históricos en que el Ciudadano Presidente de la República, Dr. Salvador Jorge Blanco, ha hecho una trascendental afirmación en el sentido de que los servicios de investigación, extensión y capacitación del sector agropecuario oficial estén a cargo de universidades del país. Este es un merecido reconocimiento que nos hace el Primer mandatario de la Nación-, porque nuestras universidades tienen el talento humano mancomunado, la capacidad investigativa, la libertad intelectual $y$ dotrinaria, de poder ejercer con objetividad científica, amplitud de miras y claro discernimiento científico, sus responsabilidades para el bienestar del sector agropecuario, de la educación del campesino y de la mayor productividad general del país. Esto lo sabe el Ciudadano Presidente, y asi lo ha proclamado en sus atinadas declaraciones.

Como Rector de la Universidad Nacional Pedro Henríquez. Ureña, le he otorgado alta prioridad, en nuestros planes institucionales de desarrollo a crear una conceptualización clara y firme para una política agropecuaria, a promover una infraestructura científica y tecnológica que sirva de apoyo eficaz para programas balanceados de investigación, educación y extensión que promueva una conciencia ciudadana favorable hacia el sector agropecuario. Incluso hemos sido los pioneros en promover la Fitopatología en nuestro planes académicos, estableciendo criterios riguiosos para formar especialistas que conozcan y manejen su campo con profesionalismo y seriedad cientítica.

Repito ahora mis palabras iniciales de admiración y respeto al trabajo que ustedes llevan a cabo, $y$ les exhorto a que recuerden que este Congreso tiene una importante misión que cumplir: ieducar a la sociedad! Nada me parece más falso que la opinión de ciertos científicos, o mejor, pseudocientíficos, que consideran que la ciencia es por definición, inaccesible a la mayoría. El primer deber del científico me parece debe consistir, por el contrario, en buscar un camino para dar a conocer alrededọr suyo lo que la misma sociedad le ha concedido como suerte, o la oportunidad, de conocer. El científico tiene la obligación moral, ante todo, de informar a los individuos del estado actual de nuestros conocimientos, para permitir a la sociedad situarse en su lugar verdadero ante los problemas y desafíos de la época. Este Congreso debe ponernos a todos en la conciencia que tiene, para nuestra propia supervivencia, el uso inteligente y correcto de la agricultura, el control eficaz de plagas y las maneras para robustecer nuestra tan amada tierra dominicana.

Quiera Dios que durante estos días ustedes aporten su inmenso grano de sal, que sirva como levadura en medio de una conciencia adormecida o ignorante. En la física se consideran algunos líquidos como en un estado de "sobrefusión": estos líquidos se hallan a una temperatura "inferior" a la que debería modificar su estado por 
fusión, pero a causa de una rara sonas, grupos o instituciones, para inercia de la naturaleza tardan en que más rápidamente de lo que solidificarse. Es suficiente, entonces, suponemos, la humanidad parta en arrojar unos pocos cristalitos apro- busca de otras realidades?

piados a la solución para que, Este Congreso es ese arranque bruscamente, ésta se solidifique por en la toma de conciencia. Son uscompleto en pocos instantes. Nues- tedes como la levadura en la masa. tra humanidad ¿No se encuentra, Adelante, pues, que todos esperamos acaso en un punto crítico análogo de su ayuda.

al de estos líquidos en "sobrefusión"? ¿No bastará, quizás, una toma de conciencia, en algunas per-

iMuchas Gracias! 